Voix et Images

voixetimages

\title{
La métrique de Gaston Miron
}

\section{Michel Lemaire}

Volume 15, numéro 3 (45), printemps 1990

\section{Gilbert La Rocque}

URI : https://id.erudit.org/iderudit/200857ar

DOI : https://doi.org/10.7202/200857ar

Aller au sommaire du numéro

\section{Éditeur(s)}

Université du Québec à Montréal

\section{ISSN}

0318-9201 (imprimé)

1705-933X (numérique)

Découvrir la revue

Citer cet article

Lemaire, M. (1990). La métrique de Gaston Miron. Voix et Images, 15(3), 396-423. https://doi.org/10.7202/200857ar d'utilisation que vous pouvez consulter en ligne.

https://apropos.erudit.org/fr/usagers/politique-dutilisation/ 


\title{
La métrique de Gaston Miron
}

\author{
par Michel Lemaire, Université d'Ottawa
}

La poésie, c'est ce qui étant dit, est.

Miron

\section{Cadre théorique}

Dès qu'on ouvre un recueil de poésie, le texte s'offre aux yeux en tant que poème par un certain nombre de signes extérieurs, par exemple le blanc inégal à droite des lignes, et par cela il impose d'emblée un certain nombre d'a priori: appartenance à l'institution littéraire, système de valeur's, code de lecture. Un poème est d'abord ce qui s'affirme comme de la poésie et ce qui est lu comme de la poésie. Les règles de la poésie traditionnelle, au niveau du vocabulaire, des figures et de la versification, constituaient un ensemble extrêmement structuré qui encadrait avec précision et rigueur le travail de l'écriture de même que le plaisir de la lecture. Lorsqu'on lit, encore aujourd'hui, un texte qui s'affiche (par exemple par le nom de son auteur ou par sa date) comme appartenant à la poésie régulière française, ce poème apporte avec lui un code de lecture èt d'appréciation tout fait; code qui permet de goûter un rythme par la prononciation des $e$ atones dans certaines circonstances précises, par exemple; ou qui permet d'apprécier un enjambement sur la césure ou un effacement complet de la césure par référence à une conception traditionnelle du vers et de la césure. Tout objet d'art est le produit d'une culture et ne peut s'apprécier que dans le cadre d'une connaissance globale de cet art et de cette culture. On a dit qu'un peintre apprenait la peinture dans les musées et non dans la nature; de même un écrivain apprend l'écriture dans les livres et non dans ses chagrins d'amour. Et ceci est particulièrement vrai de la poésie 1 .

Et c'est, je crois, toujours vrai de la poésie contemporaine. Même si celle-ci s'est libérée, par révolte ou par ignorance, de la versification traditionnelle, elle demeure largement tributaire des siècles

1 Gaston Miron: Il n'y a pas de génération spontanée dans l'écriture. Les formes engendrent de nouvelles formes. Par le nombre, le rythme et les groupes de mots, je renoue avec toute la langue. (Claude Filteau, l'Homme rapaillé de Gaston Miron, Montréal/Paris, Trécarré/Pédagogie moderne Bordas, 1984, p. 125). (Épigraphe, ibid., p. 127). 
de poésie qui l'ont précédée. Notre époque a sans doute une mauvaise mémoire et a établi l'originalité, la modernité en valeurs suprêmes, elle ne peut pour autant effacer tout héritage; on ne peut se faire primitif. Au niveau formel, le mouvement le plus profond de la poésie au $X_{X}{ }^{\mathbf{e}}$ siècle est sans doute celui d'une libération de l'écriture. Par là le poète est un des prophètes de ce siècle mettant en avant l'individualité du créateur, sa totale liberté, pour aboutir à la liberté, à l'ouverture de l'œuvre même. Cet enrichissement indéniable, cependant, s'est réalisé au détriment de la communication. Le code poétique traditionnel était un carcan, mais il avait l'avantage d'être universel (dans l'univers littéraire francophone).

Une œuvre poétique, de nos jours, s'offre au lecteur, dans une bonne mesure, solitaire et dénudée. À lui de savoir la prendre et la goûter. Il le fera à partir de ce qu'il est, bien sûr, de ses choix et de sa culture, et par là la lecture est création de l'œuvre; il le fera ensuite en replaçant l'œuvre, si cela est possible, dans un certain contexte littéraire; il le fera enfin en extrayant du texte même son code de lecture spécifique. On a dit qu'un alexandrin, par exemple, n'existait que dans la comparaison avec les autres vers appelés alexandrins; que dans le contexte d'une tradition poétique. Aujourd'hui, lorsqu'un poème nous arrive sans étiquette, sa lecture seule nous permettra d'y déterminer ce qui relève de telle ou telle conception de la poésie. Ainsi, une bonne part de la poésie actuelle, pour s'être libérée de la versification traditionnelle, n'en conserve pas moins comme valeur littéraire, une conception musicale du langage poétique, fondée sur le rythme du vers, l'harmonie des sonorités. Mais en l'absence de règles de versification contraignantes, l'évaluation de ces facteurs devra se faire à l'aide de critères tirés de l'œuvre elle-même. Si un sonnet, par exemple, pouvait être jugé en fonction de son adéquation à, ou de son écart par rapport à des règles préexistantes à lui-même, les règles dé jugement d'un poème moderne doivent, en grande part, être inventées au fil de la lecture ou du moins être adaptées au texte considéré.

Si je me propose d'étudier ici la technique du vers dans l'œuvre poétique de Gaston Miron, c'est qu'à la lecture de l'Homme rapaillé, ainsi qu'à l'audition de poèmes dits par Miron, s'est développé en moi le sentiment que cette œuvre relevait d'une conception musicale du langage poétique. Et que si cette œuvre, du moins dans sa majorité, ne se référait pas explicitement au code de la versification régulière française, néanmoins sa lecture suggérait-elle des références à ce code (en particulier une conception du vers en tant que structure rythmique régulière et close). L'hypothèse que je désire vérifier est donc celle de la relation entre l'œuvre de Miron et la versification régulière: il s'agit d'étudier dans quelle mesure cette œuvre reprend des concepts métriques traditionnels, dans quelle mesure elle s'en écarte pour s'inventer des formes nouvelles. Cette analyse se fera sans prendre en considération la connaissance que le poète peut affirmer (ou nier) 
posséder du code de la versification traditionnelle. La question de la conscience que le poète peut avoir des procédés qu'il utilise est passionnante mais secondaire (c'est-à-dire qu'elle sera traitée en second lieu). Un auteur peut ne pas se souvenir consciemment d'une partie de la culture sur laquelle est fondée sa poétique. Car si toute poétique trouve sa source dans la poésie du passé (pour la prolonger ou s'en écarter), la poétique d'une grande œuvre est devenue tellement personnalisée que ses sources historiques (dans la vie du poète) ont pu s'effacer. Ici comme ailleurs, c'est donc le texte qui doit parler d'abord.

Dans le contexte large de la poésie moderne, caractérisée par la diversité des vers et une préoccupation tout à fait facultative des questions de versification, le sentiment né, à la lecture de l'œuvre de Miron, de son caractère musical doit provenir d'une présence très marquée de signes métriques. Abstraction faite des autres caractéristiques du langage poétique et en rappelant à nouveau que le vers n'est pas la poésie, le vers se définit avant tout comme une structure rythmique: La condition nécessaire et suffisante dé l'existence du vers, c'est le rythme. S'il ne s'impose pas, il faut le chercher. ${ }^{2}$ D'où provient, par exemple, l'impression d'entendre parfois un alexandrin dans un texte de prose? Ce n'est évidemment pas le simple nombre de douze syllabes, nombre qui se retrouve partout. On se souvient, d'autre part, de l'expérience consistant à lire à des auditeurs un texte découpé en segments de douze syllabes: ceux-ci n'y reconnaissaient pas pour autant des vers alexandrins. Le sentiment du vers ne provient pas de la perception d'un certain nombre de syllabes (perception d'ailleurs difficile), mais de celle d'un rythme régulier. C'est un rythme, plus ou moins régulier, que l'on entend d'abord à écouter Gaston Miron réciter un de ses poèmes ou à le lire soi-même.

Une définition simple et générale du rythme en fait l'alternance d'éléments marqués et d'éléments non marqués. En musique, de temps forts et de temps faibles. Dans le langage, de syllabes accentuées et de syllabes non accentuées. Cette alternance, plus ou moins régulière dans la prose, est régularisée dans la versification traditionnelle par des règles précises qui font ainsi que l'alexandrin se définit, plutôt que comme une somme de douze syllabes, comme une structure rythmique 6/6 marquée par deux accents toniques fixes en sixième et douzième positions: à la césure et à la fin du vers. Soit, si le 0 indique un temps non marqué et le 1 un temps marqué (une syllabe accentuée):

\section{$\begin{array}{lllllllllllll}0 & 0 & 0 & 0 & 0 & 1 & 0 & 0 & 0 & 0 & 0 & 1 .\end{array}$.}

C'est la répétition de cette structure rythmique stricte qui donne le sentiment du vers alexandrin et les exceptions à cette norme (par exemple le trimètre romantique) ne se perçoivent auditivement comme

2 Jean Mazaleyrat, Éléments de métrique française, Paris, A. Colin, 1974, p. 57. 
vers qu'en tant qu'écart par rapport à elle. Et étant donné que les groupes rythmiques du français comprennent généralement deux, trois ou quatre syllabes, chacun des hémistiches ainsi délimité sera à son tour divisé, la plupart du temps, en deux groupes rythmiques (que l'on peut identifier à des mesures) par un accent tonique de position variable. Les deux accents fixes assurant la régularité du vers, les deux accents mobiles sa variété. On en arrive ainsi à définir l'alexandrin régulier (pris ici comme exemple) ainsi qu'une structure rythmique complexe de quatre mesures; ce qu'on représente habituellement par des schémas du type:

\section{$2 / 4 / / 2 / 4(010001010001)$ \\ ou $3 / 3 / / 3 / 3(001001001001)$.}

Ceci n'est pas nouveau et Albert Thibaudet décrivait déjà l'alexandrin comme un tétramètre. Mais je voudrais insister sur ce qui me semble être la pérennité de cette structure rythmique dans la poésie moderne (et, on le verra, spécialement dans l'œuvre de Miron). La culture poétique de l'écrivain et du lecteur actuels, et en particulier l'empreinte indélébile de l'école et de ses poèmes appris par cœur, font, je crois, que ce rythme pair fondé sur le groupe de six syllabes (et à la limite l'alexandrin "absolu " $3 / 3 / / 3 / 3$ ) apparait toujours "naturellement" le meilleur, le plus harmonieux de la poésie versifiée. Je ne pense pas nécessaire d'opposer ici une conception syllabique et une conception rythmique du vers; car si c'est effectivement le rythme qui donne le sentiment du vers, la mesure, en français, est bien constituée d'un certain nombre de syllabes. Les hypothèses d'une égalité de durée des mesures (indépendante du nombre de syllabes) ou de l'allongement compensatoire de certaines syllabes (pour les faire équivaloir à deux syllabes) ne me paraissent pas, pour le moins, généralisables. Je vois une preuve de cette base syllabique du vers français dans le fait qu'instinctivement, à la lecture, on va "avaler" un $e$ atone ou ajouter un $e$ épenthétique dans le but non d'égaliser des durées de vers mais d'obtenir le nombre de syllabes qui semble nécessaire. L'analyse de la versification d'une œuvre en "vers libres " telle celle de Gaston Miron nous replace donc devant deux problèmes classiques dans le domaine: la détermination des groupes rythmiques et celle des syllabes qui comptent effectivement dans la mesure du vers, ces syllabes qu'on nomme traditionnellement en français les "pieds".

Comme corpus d'analyse, plusieurs études, depuis le début du siècle, se fondent sur l'enregistrement de la lecture de poèmes par un lecteur choisi ou par le poète lui-même, ainsi que sur la transcription sous forme d'oscillogrammes de cet enregistrement. Ce choix mérite discussion. Les instruments du phonéticien ont apporté des réponses nombreuses et précises parce qu'ils permettent de voir et de mesurer les sons du langage: les groupes rythmiques, ainsi, y prennent une évidence indéniable. Mais d'un point de vue littéraire, cette approche 
a bien sûr ses limites. Car il ne faut pas oublier que ce qui est analysé alors n'est pas le texte lui-même mais une certaine lecture, une certaine interprétation de ce texte. Or cette. interprétation, que ce soit celle d'un comédien ou celle du poète, peut, pour diverses raisons, être discutable, ne pas répondre par exemple à ce qu'une analyse littéraire du texte pourrait suggérer. On sait que l'auteur n'est pas toujours le lecteur de son œuvre le plus intéressant. Toutefois le cas de Miron est particulier et pose cette question de façon plus impérieuse. Gaston Miron est reconnu pour ses talents de conteur et d'interprète de son œuvre. Une analyse métrique de celle-ci ne devrait-elle pas se baser sur un enregistrement des récitals de l'auteur? Dans la mesure où l'Homme rapaillé trouve son origine dans un travail oral du texte et est lié, presque intrinsèquement, à la parole sociale du poète, ce type d'analyse sera ici plus important qu'ailleurs. Cependant, dans un parti pris littéraire, je choisis, pour les raisons invoquées précédemment, de faire précéder cette étude d'une analyse du texte seul, quitte ensuite à confronter cette analyse purement textuelle à une autre plus vaste, à les enrichir ou à les contredire l'une par l'autre.

Il me semble intéressant, en particulier, de tenter de déterminer dans quelle mesure un poème contemporain peut, de lui seul et indépendamment de telle ou telle interprétation (au sens théâtral du terme), imposer ou proposer son ou ses rythmes de lecture. Mais cette possibilité me paraît immédiatement confrontée à deux caractéristiques de ce texte, deux libertés qu'il s'est données: son ouverture syntaxique, l'absence de règles de prononciation. Prenons d'abord la première de ces questions. Si l'absence de ponctuation, assez générale dans la poésie contemporaine, est autre chose qu'un artifice de présentation, elle se conjugue avec une utilisation particulière de la syntaxe (indéterminations diverses) pour ouvrir le texte (au-delà de l'ouverture déjà connue des images) et imposer, en plus d'une polysémie, une pluralité de lectures. Et si lire un tel texte ne consiste pas simplement à rétablir une ponctuation qu'un auteur facétieux aurait effacée, à tracer une direction de signification, comment peut-on concilier cette multiplicité avec la recherche du rythme du texte? Peut-il exister plusieurs rythmes en même temps (non pas potentiels mais réalisés)? Si un mot peut porter plusieurs sens, une phrase peut-elle offrir, en même temps, plusieurs musiques? Je ne crois pas, et il me semble alors que la lecture ne peut éviter d'être réductrice.

La seconde question est celle de la lecture du texte, au sens de la prononciation des phonèmes qui le composent, étant donné l'absence de règles semblables à celles qui régissaient la lecture des vers réguliers. Ces règles (concernant la prononciation des $e$ atones, les diérèses) s'étaient imposées pour contrôler les aléas de la prononciation usuelle (prosaïque) du français. La poésie du $\mathrm{XX}^{\mathrm{e}}$ siècle, en majorité, les a rejetées en revendiquant la liberté et le naturel de la langue parlée. La difficulté naît de la contradiction entre les caractéristiques 
de cette langue parlée, qui vise l'efficacité, la rapidité de la communication et pour cela n'hésite pas à éliminer ou à fusionner plusieurs phonèmes, et celles de la langue poétique pour laquelle ce principe d'économie phonétique n'existe pas. Si on admet que le langage poétique se fonde sur une utilisation totale des signes linguistiques, signes non pas réduits à leur signifié ainsi que dans la prose courante mais pris comme objets sonores charriant la diversité de leurs connotations, la meilleure lecture sera celle qui mettra le mieux en évidence cette richesse de l'imaginaire et des sonorités. Ce sera donc une lecture attentive à relever chaque sonorité, chaque mot porteur de signification. La poésie est musique; pour réaliser le poème, cette musique doit être entendue et donc d'abord émise par les organes phonateurs (même dans une lecture murmurée). Toutefois le ton soutenu qui résulte d'une telle lecture ne peut plus, à notre époque, s'appuyer sur des diérèses artificielles ou sur la prononciation systématique des $e$ atones. Il y a un choix à faire, une interprétation à déterminer; ainsi, les tentatives pour créer un système guidant cette lecture (comme les apostrophes pour remplacer les $e$ devenus muets) ayant échoué, chaque vers devient un problème rythmique à résoudre.

On peut cependant essayer d'établir un certain nombre dé critères sur lesquels s'appuieront les choix de lecture. On s'accordera, je crois, sur le principe d'une prononciation soutenue, distinctive mais naturelle. Les synérèses et diérèses seront donc celles de la langue parlée (sauf exceptions à justifier stylistiquement). Le cas des $e$ atones, lui, est beaucoup plus complexe. Distinguons d'abord les $e$ qui, dans la langue d'aujourd'hui, peuvent encore se prononcer de ceux qui ne le peuvent plus: Le $e$ final d'un groupe syntaxique ne peut plus, en français moderne, se faire entendre (sa prononciation, dans un certain 'style de chanson, fait sourire); il tombera donc, dans le vers libre, naturellement et systématiquement; et c'est sa conservation qui devra être justifiée. Par exemple, dans le groupe la petite fille placé en fin de proposition, l'apocope du $e$ final de fille sera la norme. Les $e$ de petite, situés à l'intérieur du groupe dans un mot signifiant, eux se prononceront normalement et c'est leur chute qui devra être justifiée (par exemple pour des raisons de métrique). Cette première règle de l'apocope des $e$ finals fait que, la plupart du temps, les $e$ situés à la fin d'une mesure et en particulier les $e$ situés à la césure ne seront pas prononcés. Cette renaissance de ce qu'on appelle traditionnellement la césure épique est une caractéristique importante du vers moderne qui a déjà été remarquée par la critique ${ }^{3}$.

L'établissement de règles de prononciation des $e$ à l'intérieur des groupes syntaxiques est plus délicat. Il s'agit bien sûr des $e$ entre consonnes, l'élision des $e$ précédés ou suivis de voyelles se faisant

3 Cf. Henri Morier, le Rythme du vers libre symboliste, Genève, Presses académiques, 1943-1944, 3 vol., vol. III, p. 41 ; et Jean Mazaleyrat, op. cit., p. 68. 
naturellement, et leur conservation devant se justifier. Des analyses de Henri Morier dans le Rythme du vers libre symboliste et dans l'article " $\mathrm{E}$ caduc» de son Dictionnaire de poétique et de rhétorique, je tirerais les principes suivants:

- préférence de l'apocope (chute du $e$ à la fin d'un mot) à la syncope (chute d'un $e$ interne) et en particulier de l'apocope dans les mots-outils à la syncope dans les mots chargés de sens ${ }^{4}$;

- règle des trois consonnes: le contact possible de trois consonnes empêche, en général, la chute du $e^{5}$;

- règle de l'alternance des apocopes dans une suite de syllabes contenant des $e$ atones ${ }^{6}$.

Tous ces choix de lecture doivent se subordonner à un principe supérieur qui est celui de la beauté sonore du vers considéré comme une unité structurée. Pour ce faire, Mazaleyrat propose de se laisser guider par les soucis suivants: D'abord réaliser les structures rythmiques latentes. [...] En second lieu, marquer les articulations du vers? Toutefois la mise en pratique de ces conseils doit s'effectuer avec beaucoup de doigté. Réaliser les structures rythmiques latentes présuppose qu'elles existent, et qu'elles sont unes et non multiples. Maints vers modernes laissent perplexe à ce niveau et l'on peut se demander si, souvent, le lecteur ne plaque pas sur un vers un rythme qui n'y était pas si «latent». La culture du lecteur peut l'amener, par le jeu des apocopes, à "réaliser" systématiquement les vers devant ses yeux en structures paires et symétriques; tout vers ainsi lu (ou torturé) deviendra, par la force des habitudes, hexa-, octo-, déca- ou dodécasyllabique. Et si le rythme naturel du poète était impair? Ou si celui-ci avait cherché à composer des vers volontairement «faux "? L'alexandrin coupé 5/7, par exemple, n'est pas rare au $\mathrm{XX}^{\mathbf{e}}$ siècle; le lecteur ne se tromperait-il pas qui s'efforcerait de le rendre symétrique? Encore une fois, il faut donc se fier au texte: c'est le contexte proche (le poème lui-même par exemple) qui permettra de déterminer la nature du rythme de chaque vers. Mais, comme le dit bien Mazaleyrat, ces soucis de lecture visent à réaliser les structures rythmiques du vers et non à atteindre un nombre, quelconque ou déterminé, de syllabes, puisque ce nombre importe moins que la distribution des syllabes en groupes rythmiques.

4 Est universellement caduc l'e atone des mots usuels "une», "elle», "celle", "cette», "même", "toute", "comme". (Henri Morier, Dictionnaire de poétique et de rhétorique, Paris, P.U.F., 1981, p. 149). Cf. aussi le Rythme du vers libre symboliste, op. cit., vol. III, p. 41.

5 . H. Morier, Dictionnaire de poétique et de rhétorique, p. 152. Dans la versification régulière, il suffit de deux consonnes (règle des deux consonnes).

$6 \quad$ Ibid., p. 157.

7 J. Mazaleyrat, op. cit., p. 70. Et, p. 71 : un troisieme imperatif à observer dans le choix à faire entre les amuïssements possibles: donner au vers l'expressivité optimale. 
Il est sûr, d'autre part, que la prononciation ou la nonprononciation des $e$ atones modifie l'harmonie du vers. On a étudié les particularités du vers français traditionnel résultant de la multiplicité de ces $e$, tant pour ce qui est de l'équilibre entre voyelles et consonnes que pour la douceur et le caractère feutré de son harmonie; inversement, la vigueur et le dynamisme du vers moderne résultant des heurts de consonnes sont évidents. Cependant cette différence au niveau harmonique me semble moins essentielle que les modifications de rythme que provoquent la prononciation ou l'amuïssement des $e$ dans le vers libre. Le rythme s'établissant par l'alternance des syllabes accentuées et non accentuées, la prononciation des $e$ atones a pour effet de multiplier les syllabes non accentuées (temps non marqués) et ainsi de modifier le rapport temps marqués/temps non marqués. À la limite, on peut dire que la fonction rythmique des syllabes non accentuées est d'espacer, de distribuer les temps marqués.

Ces temps marqués sont constitués dans le langage, on le sait, par les syllabes accentuées. Du point de vue de la versification, la nature des accents, leur typologie selon des paramètres phonétiques ou stylistiques, ne sont pas pertinentes. Seule importe la distribution de ces accents dans la chaîne de syllabes qui forme la phrase; car seule importe la délimitation des mesures par l'accent, puis l'organisation des mesures en un mètre régulier ou en un rythme plus flexible de la poésie moderne. Toutefois la question de la nature des accents réapparaitt en raison de la diversité des analyses accentuelles: la complexité théorique de ce problème des accents en linguistique fait sans doute que le découpage des vers en mesures, pratique qui devrait être assez simple puisque instrumentale, varie passablement suivant les critiques, pour aboutir parfois à une prolifération incongrue des accents et donc des mesures; si on place des accents sur toutes les syllabes, il n'y a plus de rythme puisque le rythme provient de l'alternance entre temps marqués et non marqués. Il est indispensable, selon moi, de distinguer rigoureusement les accents rythmiques des accentuations qui proviennent de l'interprétation (au sens théâtral) du vers: il est possible, à la lecture, de mettre en évidence, d'une manière ou d'une autre, chacune des syllabes (par ce que la phonétique appelle des accents affectifs, intellectuels ou oratoires); cette mise en évidence ne constitue pas pour autant un rythme. Fondamentalement, le rythme du vers résulte de la distribution dans la phrase de ce que Morier nomme l'accent horizontal, temporel ou oxytonique ${ }^{8}$. Cet accent provient de l'allongement de la dernière syllabe (ou de l'avant-dernière si la dernière contient un $e$ atone) de chaque groupe syntaxique. C'est le seul accent qui importe du point de vue de la versification. Les autres

8 En français, l'accent temporel règne en maître dans la diction du vers scande. (H. Morier, Dictionnaire de poétique et de rhétorique, p. 22) Seul l'accent temporel règle à la fois rythme et signification. (Ibid., p. 38) 
(sauf exceptions que nous allons voir) doivent être considérés comme non métriques, c'est-à-dire n'entrant pas dans la constitution du mètre: L'établissement en une même analyse de tous les accents (métriques et non métriques) d'un vers, en une tentative d'aperception globale du rythme, amène, à mon avis, plus de confusion que d'éclaircissement.

Dans la parole, néanmoins, le découpage de la phrase ne se fait pas toujours d'une manière aussi stricte que dans la grammaire. On aura tendance à unir deux syntagmes brefs, abolissant certains accents. Inversement, on divisera un groupe long (plus de quatre syllabes) spécialement pour mettre en évidence un mot important; cet accent supplémentaire pourra se définir soit comme une réactivation d'un accent tonique effacé par la fusion syntagmatique, soit comme un accent contre-tonique (qui frappe la seconde syllabe précédant la syllabe accentuée d'un mot très long). Par le positionnement de ces accents secondaires, la division du vers en mesures pourra, avec raison, varier suivant l'interprétation.

J'ajouterai à ces accents linguistiques des accents que j'appellerai "métriques", c'est-à-dire suscités par la dynamique du vers. J'en distinguerai deux sortes: l'accent terminal et l'accent intérieur. Lorsqu'il y a concordance entre le découpage métrique et le découpage syntaxique de la phrase, un accent tonique marque la dernière syllabe non caduque du vers (c'est la norme); lorsqu'il y a discordance, dans les cas de rejet ou d'enjambement, une syllabe, non accentuée naturellement, peut se trouver en fin de vers. Si l'on considère le vers comme une structure rythmique autonome, sa dernière syllabe doit être accentuée afin d'indiquer la clôture du vers, ce qui conduit parfois à placer un accent tonique sur un mot qui sans cela n'en porterait pas. C'est le fait d'" enjamber" cet accent et la pause qui le suit qui permet d'entendre l'effet stylistique du rejet ou de l'enjambement. Deuxièmement, je ferai remarquer certains accents suscités par la régularité métrique d'un poème. L'alexandrin classique foisonne de ces accents intérieurs provoqués par la régularité de métronome du découpage rythmique plus que par la langue. L'accent que l'on est entraîné à placer en sixième position d'un alexandrin pour faire sentir un enjambement sur la césure participe de l'un et l'autre de ces deux types.

Afin d'illustrer le modèle d'analyse établi dans les pages précédentes, j'aimerais enfin prendre comme exemple un vers de Miron tiré d' "Héritage de la tristesse" ${ }^{9}$. Simplement parce que ce vers a été étudié en détail par Claude Filteau dans son livre sur l'Homme rapaillé de Gaston Miron et que la discussion de ses conclusions

9 Gaston Miron, l'Homme rapaillé, Montréal, Presses de l'Université de Montréal, 1970, p. 49. 
me paraît propre à l'intelligence des questions de versification impliquées. Filteau écrit: Prenons un vers de Miron et appliquons-lui les règles de la prosodie classique de manière que les coupes accentuelles correspondent à certaines coupes syllabiques:

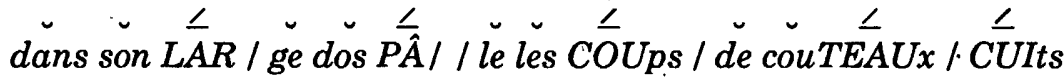
3 3 3 3

Nous avons là une sorte d'alexandrin de treize pieds, mais avec des coupes accentuelles régulières 3-3-3-3-1 et un effet de césure après la sixième syllabe. ${ }^{10}$

Puis Filteau propose les scansions suivantes:

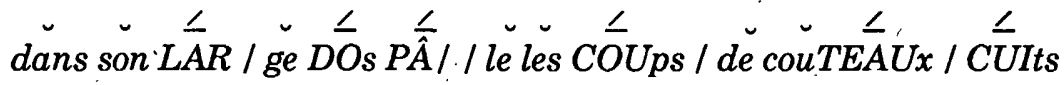
et

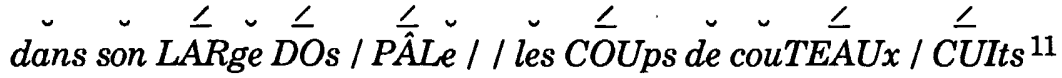

Les problèmes que soulèvent ces scansions, pour Filteau lui-même, me semblent découler d'une part d'une confusion entre découpage syntaxique et découpage métrique et d'autre part d'une conception très floue de la nature du rythme poétique (méprise entre rythme du vers et harmonie consonantique). Dans le premier schéma, pourquoi présenter différemment dos pâle et couteaux cuits sinon pour respecter un rythme en anapestes qui n'a pas été justifié ? Dans les second et troisième schémas, à quoi correspondent, en français, ces mesures contenant deux accents toniques: /ge dós pá / /; dans son lárge dós; les coúps de couteaúx? Comment expliquer que le $e$ atone de pâle ne compte plus dans le troisième schéma? Dire que peu importe que le vers ait douze ou treize syllabes ${ }^{12}$ ne fait qu'esquiver la question. Et le troisième schéma n'est finalement qu'une illustration empirique d'un sentiment juste, celui de la symétrie du vers.

Étáblissons d'abord le nombre de pieds dans ce vers car, quoi qu'en dise Filteau, ce nombre a son importance (du moins méthodologique). La question se pose à propos des trois $e$ atones de large, pâle et de. Le $e$ de pâle, final de groupe à la césure, tombe normalement (c'est notre première règle, celle de la césure épique; la césure enjambante serait ici bien peu euphonique). Les $e$ de large et de de doivent être traités de la même manière pour respecter la symétrié évidente du vers. Ce qui nous laisse avec deux possibilités:

10 Claude Filteau, op. cit., p. 11. Prosodie classique avec une césure enjambante?

11 Ibid., p. 12.

12 Ibid., p. 12. 


$$
\text { ○13 }
$$

dans son large dos pâle les coups de couteaux cuits

$$
\begin{array}{llllllllllllll}
1 & 2 & 3 & 4 & 5 & 6 & / / & 7 & 8 & 9 & 10 & 11 & 12
\end{array}
$$

dans son large dos pâle les coups de couteaux cuits
$\begin{array}{lll}1 & 2 & 3\end{array}$
$\begin{array}{lllll}4 & 5 & / / & 6 & 7\end{array}$
$\begin{array}{lll}8 & 9 & 10\end{array}$

La première versión (alexandrin) me paraît plus en accord avec le contexte rythmique du poème fondé sur des vers très longs; la seconde (décasyllabe) est cependant intéressante par sa vigueur phonétique (heurt des consonnes) en accord avec le sémantisme.

La détermination des mesures par le positionnement des accents toniques horizontaux, d'autre part, est un peu délicate dans ce cas-ci car les groupes syntaxiques se terminent par des adjectifs monosyllabiques. Les trois syllabes de chaque hémistiche qui peuvent pórter un accent tonique, peuvent toutefois difficilement être allongées exactement de la même manière; ce qu'admet Filteau mais ce dont il ne tire pas les conséquences. Les accents toniques de pâle et de cuits, en fin de groupes syntaxiques, ne posent pas de problème; ce sont les autres. Je crois que la lecture peut hésiter entre deux structures rythmiques:

dans son large dos pâle les coups de couteaux cuits

$$
\begin{array}{lllllllllllll}
1 & 2 & 3 / 4 & 5 & 6 & / / & 1 & 2 & / & 3 & 4 & 5 & 6
\end{array}
$$

l'allongement de dos et -teaux s'effaçant. Et:

dans son large dos pâle les coups de couteaux cuits

$$
\begin{array}{llllllllllll}
1 & 2 & 3 & 4 & 5 & / / & 1 & 2 & 3 & 4 & / & 5
\end{array}
$$

l'allongement de dos et -teaux provoquant l'effacement des accents de large et coups de même que l'apocope des $e$ de large et de. Cette seconde lecture est expressive mais la mise en relief qu'elle provoque de pâle et cuits est-elle justifiée par l'importance sémantique de ces mots? J'en doute, c'est pourquoi je préfêre la lecture précédente, en alexandrin régulier scandé $3 / 3 / / 2 / 4$ :

dans son large dos pâle les coups de couteaux cuits

$$
\begin{array}{llllllllllllll}
1 & 2 & 3 / 1 & 2 & 3 & / / & 1 & 2 & / & 1 & 2 & 3 & 4
\end{array}
$$

Cette scansion est une lecture, c'est-à-dire un choix personnel, mais j'espère l'avoir ici assez précisément et assez clairement étayée. Elle

13 J'indique par ce signe la chute du $e$ atone. 
illustre la démarche que je me propose de suivre dans mon analyse de l'œuvre de Miron.

\section{L'Homme rapaillé 14}

Lq poésie est rythme et images («Ma bibliothèque idéale», p. 105), rappelle Gaston Miron. Toutefois, lorsqu'on s'est penché sur son œuvre, on s'est beaucoup moins intéressé au rythme qu'aux images. Pourtant, dans ses "Notes sur le non-poème et le poème", Miron définit d'abord sa poésie comme souffle, comme rythme de vie:

\section{mon poème}

entre haleine et syncopes

ce faible souffle phénix d'un homme cerné d'irréel (p. 125)

Ce qui me frappe dans les premiers poèmes de l'Homme rapaillé, c'est précisément la difficulté du poète à respirer de son propre souffle, à atteindre cette liberté fondamentale d'un homme et d'un artiste de parler à son rythme et avec ses mots. Le poème devra s'arracher à la misère du "non-poème", mais aussi se détacher de fausses richesses empruntées, pour inventer sa "voix", rythme et images:

je ne veux pas me laisser enfermer

dans les gagnages $d u$ poème, piégé fou raide

mais que le poème soit le chemin des hommes ${ }^{15}$

Pierre Nepveu remarque que les premiers poèmes de Miron peuvent se diviser en deux groupes: d'une part les textes les plus familiers, les plus proches d'une pure reproduction de la parole, et d'autre part des poèmes au ton très littéraire ${ }^{16}$ qui font appel à tout l'arsenal de la versification régulière. Ce n'est que progressivement que Miron réussira à abolir cette dichotomie ${ }^{17}$, à se dégager de ces influences ${ }^{18}$, pour forger le vers de ses grandes suites poétiques à partir de "La marche à l'amour».

Dans les "Premiers poèmes", le rythme me paraît en général manquer singulièrement d'intérêt: découpage en vers directement calqué sur les groupes syntaxiques et n'apportant que peu de choses au poème, textes progressant par énumérations et dont le rythme est

14 Désormais, les indications de page seules, entre parenthèses, renverront à l'édition des Presses de l'Université de Montréal de l'Homme rapaillé.

15. Gaston Miron, Courtepointes, Ottawa, Éditions de l'Université d'Ottawa, 1975, p. 44-45. Je cite l'Homme rapaillé et Courtepointes d'après les éditions canadiennes puisque ce sont ici les plus courantes. Mais j'étudierai les variantes les plus intéressantes apportées par l'édition de 1981 (Paris, François Maspero).

16 Pierre Nepveu, les Mots à l'écoute, Québec, les Presses de l'université Laval, 1979, p. 127.

17 Ibid.

18 C'est le nouveau titre de la section “Premiers poèmes" dans l'édition Maspero. 
répétitif, enchaînements de vers laborieux, au rythme incertain. Ici le ton se veut familier et le poète utilise ce procédé typique de la poésie populaire qu'est l'apostrophe signalant l'apocope, procédé d'ailleurs qu'il n'emploiera plus dans l'avenir:

il s'est mis à s'tasser

il s'est mis à s'manger

on n'a jamais vu ça

un homme qui se mange

("Fait divers », p. $28^{19}$ )

Ailleurs au contraire le ton rappelle celui de la "grande" poésie lyrique et chante l'amour dans un vocabulaire recherché. Ces poèmes font explicitement référence à la tradition poétique française (Mallarmé, Valéry) et utilisent les ressources de la versification classique. On a l'impression que Miron fait ses gammes de versificateur. Plusieurs poèmes présentent ainsi un encadrement métrique tout à fait régulier; jetons-y un coup d'œil pour commencer. Le poème "Semaines" se compose de quatre quatrains de vers de six syllabes (en comptant les pieds selon les règles traditionnelles); les rimes (assez pauvres) en sont croisées pour les trois premiers quatrains et embrassées pour le dernier (Miron ne respecte pas la règle du nombre singulier/pluriel des rimes, ni, dans les deux derniers quatrains, celle de l'alternance du genre masculin/ féminin des rimes). La version de l'édition Maspero de ce poème, intitulée "Chanson", apporte deux variantes qui prouvent le respect qu'a Miron des règles traditionnelles. Dans le troisième quatrain:

sept jours comme des flûtes

les balcons qui colportent

le front blême qui bute

sur le seuil muet des portes

("Semaines", p. 13),

les deux derniers vers deviennent:

le front las qui se bute

au seuil muet des portes 20

Le poème "Cantique des horizons", sous-titré sur un ton faussement valéryen (p. 17), est composé lui aussi de quatrains d'hexasyllabes; les rimes, embrassées pour tous les quatrains sauf le dernier où elles - sont croisées, respectent la règle de l'alternance des genres mais non celle du nombre. Le ton précieux du poème est accru par la reprise de certains vers. Enfin le poème "Pour retrouver le monde et l'amour" (p. 19) présente des sizains d'alexandrins coupés régulièrement 6/6. Les rimes plates suivent les mêmes règles que précédemment.

19 Le vers est donc ici "officiellement " un hexasyllabe.

20 Gaston Miron, "Chanson", l'Homme rapaillé, Paris, François Maspero, 1981, p. 14. La synérèse de muet était discutable. 
On ne peut dire que le recours à la versification régulière apporte beaucoup à ces poèmes: le rythme en est scolaire, les rimes assez pauvres et peu intéressantes. Le pastiche de Mallarmé, en vers irréguliers, est plus amusant; Miron y utilise l'assonance plutôt que la rime ("Corolles ô fleurs", p. 18). D'autres poèmes débutent dans un patron assez régulier pour s'en échapper par la suite: les quatre premiers vers de "Mon bel amour" riment entre eux (p. 9); il en est de même pour la première strophe de "Ce corps noueux" (p. 11). Le poème «Déclaration" (p. 30) offre une suite de quatrains non rimés au rythme très proche de l'alexandrin en particulier au début. Mais tous ces textes démontrent l'intérêt de Miron pour la versification et sa bonne connaissance des règles traditionnelles du vers français. Ils démontrent aussi la nécessité où il se trouvait de se libérer de ce cadre trop étroit pour lui. Le dernier poème de cette section, "La route que nous suivons" (p. 31), effectue cette libération de manière éclatante: il est, pour l'essentiel, composé de vers non réguliers, non rimés, de douze à vingt syllabes. Ces longs vers sinueux, généralement découpés en trois parties, contrastent nettemént par leur fluidité avec ceux que nous venons de voir. Cette libération, cependant, était peut-être trop extrême pour Miron qui privilégiera toujours un vers très structuré: la variante de ce poème qu'on découvre dans l'édition Maspero, nous présente en effet une réorganisation complète du rythme. Les grands vers sont démembrés, le rythme est resserré autour de celui de l'alexandrin: pour ce faire, Miron réduit une syntaxe trop prosaïque et redécoupe son texte en hémistiches mieux délimités. Ainsi la strophe:

soudain je suis debout contre l'air égratigné de mouches à feu

je suis droit à l'écoute comme un fil à plomb

je comprends que nous ne serons plus jamais des hommes si nos yeux se vident

devient:

soudain contre l'air égratigné de mouches à feu

je fus debout dans le noir du Bouclier

droit à l'écoute comme fil à plomb à la ronde

nous ne serons jamais plus des hommes

si nos yeux se vident de leur mémoire

(l'Homme rapaillé, édition Maspero, p. 32)

Avec "La marche à l'amour", Miron apparait pleinement en possession de l'instrument poétique qui lui permettra de réaliser son œuvre. Des éléments réguliers demeurent et demeureront par la suite - tel le quatrain d'alexandrins approximatifs -, mais il a réussi à prendre ses distances de la versification régulière, tout en s'appuyant sur les acquis qu'il en avait retirés, pour élaborer le vers personnel que nous allons maintenant étudier. Miron conserve de la versification traditionnelle le concept du vers comme unité rythmique close sur elle-même. Sa phrase 
poétique est une période d'une belle ampleur qui se déroule sur plusieurs vers; la syntaxe, relativement légère, est pourtant très nette et structure la phrase de manière évidente en groupes bien délimités; la concordance entre ce découpage syntaxique et le découpage rythmique proposé par le vers clôt chaque vers sur lui-même en une unité sémantico-rythmique clairement perceptible. Mais ce vers s'est délesté d'autres règles traditionnelles: il est non rimé, il n'est pas toujours de la même longueur, les $e$ atones n'y semblent pas systématiquement prononcés selon la loi des deux consonnes. Dans sa forme la plus fréquente, ce vers paraît être d'une douzaine de syllabes ${ }^{21}$, c'est le grand vers de Miron, celui sur lequel se développent la majorité de ses poèmes et spécialement les grandes suites qui constituent le cœur de l'Homme rapaillé.

Comment ce vers se compare-t-il avec l'alexandrin pratiqué précédemment? Un certain nombre de vers de Miron peuvent effectivement se lire comme des alexandrins réguliers: fermés sur eux-mêmes, nettement divisés par une césure au sixième pied, ils offrent un compte exact de douze pieds.

un visage enneigé de hasards et de fruits,

("La marche à l'amour", p. 37)

tu es ma chance ouverte et mon encerclement

(p. 38)

me voici de nouveau campé dans ta légende

(p. 41)

sont de beaux alexandrins classiques tirés de "La marche à l'amour". Mais on en retrouve des exemples dans toute l'œuvre:

cependant qu'en mes bras ma jeunesse rougeoie ("L'octobre" p. 62)

dans les sables mouvants des détresses grégaires (p. 62)

Toi qui es mon amour dans l'empan de ma vie

("Au sortir du labyrinthe», p. 72)

ma mère au cou penché sur ton chagrin d'haleine

("Art poétique», p. 79)

Et ce sont souvent les vers les plus importants, le premier ou le dernier vers d'une strophe ou d'un poème, ce sont souvent les vers les plus typiques de l'œuvre, les plus " mironiens ", qui adoptent ce rythme de l'alexandrin:

Je suis un homme simple avec des mots qui peinent

(«Avec toi », p. 45)

et parfois je me traîne et parfois je rafale...

("Après et plus tard " p. 73)

21 Il n'est pas possible de réaliser des décomptes exacts puisque ce nombre de syllabes est approximatif. 
qui donc démêlera la mort de l'avenir

(«Le camarade», Courtepointes, p. $27^{22}$ )

À côté de ces alexandrins, on découvre de nombreux vers que, par contagion je dirais, on est porté à lire comme des alexandrins. Il est nécessaire pour cela de prendre de petites libertés dans le compte des $e$ atones. Mais les règles que nous avons établies dans la première partie, nous permettent de lire des vers comme les suivants ainsi que des alexandrins simplement libérés du compte strict des $e$ atones:

j'allumerai chez toi les phares de la douceur

$\begin{array}{llllllllllll}1 & 2 & 3 & 4 / 1 & 2 & 2 / / & 2 & / & 1 & 2 & 3 & 4\end{array}$

(«La marche à l'amour», p. 39)

La mort trébuchera dans sa dernière moisson

\section{$\begin{array}{llllllllllllll}1 & 2 & / & 1 & 2 & 3 & 4 / / & 1 & 2 & 3 & 4 & / & 1 & 2\end{array}$}

("L'ombre de l'ombre», p. 80)

En particulier, la règle de la césure épique (apocope du $e$ posttonique à la césure) me paraît être prépondérante et permettre la scansion la plus «naturelle» du vers mironien:

lentement je m'affale tout au long de ma hampe

$\begin{array}{llllllllllllll}1 & 2 & 3 & 1 & 1 & 2 & 3 & / / & 1 & 2 & 3 / & 1 & 2 & 3\end{array}$.

("La marche à l'amour», p. $41^{23}$ )

22 E. Roberto, dans son ouvrage sur les Structures de l'imaginaire dans Courtepointes de Miron (Ottawa, Éditions de l'Université d'Ottawa, 1979, p. 41), donne une variante où l'on voit que ce vers n'a pas encore atteint son rythme d'alexandrin: qui démélera la mort de l'avenir

C'est le cas le plus fréquent: en général, Miron, en travaillant ses textes, accroît leur régularité rythmique. Autre exemple:

Les bulles du delire les couleurs

(Courtepointes, p. 44)

deviendra (édition Maspero, p. 53):

Les bulles du délire les couleurs débraillées

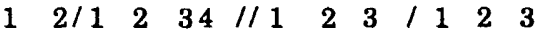

Voici cependant un exemple inverse:

nous n'irons plus mourir de langueur mon amour

le premier vers de la troisième partie de "La marche à l'amour» (p. 17), devien-

dra, dans l'édition Maspero (p. 39): nous n'irons plus mourir de langueur

l'alexandrin étant abandonné.

23 La variante de l'édition Maspero (p. 42) conserve à peu près le même rythme:

lentement je m'affale de tout mon long dans l'âme

$\begin{array}{llllllllllllll}1 & 2 & 3 & 3 & 1 & 2 & 3 & / / & 1 & 2 & 3 & 4 & / & 1\end{array}$ 
avec mon corps en cendres et mes yeux en dedans

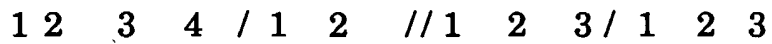

("Après et plus tard» p. 73)

la tristesse du monde luit très lasse et très basse

$\begin{array}{lllllllllllll}1 & 2 & 3 / 1 & 2 & 3 & / / & 1 & 2 & 3 & / & 1 & 2 & 3\end{array}$

(p. $73^{24}$ )

En général, l'apocope à la fin d'une ou de plusieurs mesures suffit à instituer un rythme d'alexandrin. Ainsi, l'alexandrin régulier:

ton corps tiède de pruche à mes bras pagayeurs

de "La marche à l'amour" est-il précédé de ce vers qui peut se lire sans difficulté avec le même compte de douze:

harmonica du monde lorsque tu passes et cèdes

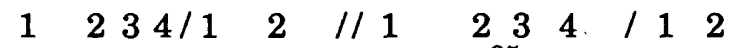

( "La marche à l'amour ", p. 39 25)

De même:

à la rétine d'eau pure dans la montagne natale

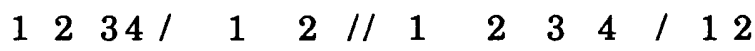

(«Le damned Canuck», p. 55)

La lecture du vers suivant - si célèbre - me paraît toutefois plus difficile:

Les poètes de ce temps montent la garde du monde

("Recours didactique", p. 61)

J'accorderais six pieds au premier hémistiche (la prononciation du $e$ de poètes entraînant une cascade de trois $e$ atones; la nonprononciation des deux autres $e$ étant trop familière):

Les poètes de ce temps

$$
\begin{array}{llllll}
1 & 23 & / & 1 & 2 & 3
\end{array}
$$

24 A-t-on au contraire des césures dans les vers suivants: puis les années m'emportent sens dessus dessous (6/6? "La marche à l'amour", p. 40)

le vieil Ossian aveugle qui chante dans les radars

(6/8 ou $6 / 6$ ? Courtepointes, p. 26)?

Ces cas sont rares.

25 La prononciation du e de passes créerait une liaison euphonique. 
Si on opte pour la symétrie du vers et qu'on accorde six pieds au second hémistiche (la symétrie pourrait aussi être obtenue en donnant sept pieds à chacun), on peut hésiter à propos des deux $e$ atones; je préfere la scansion suivante, plus conforme à la syntaxe:

Les poètes de ce temps montent la garde du monde.

$$
\begin{array}{llllllllllllll}
1 & 23 & / & 1 & 2 & 3 & / / & 1 & 2 & 3 & 4 & / & 1 & 2
\end{array}
$$

Pour.traduire cet attachement à la métrique régulière - attachement qui se double d'un écart -, Miron, rappelant le nom de Baudelaire, parle d'écho: Dans ma démarche, je recherche aussi l'écho de formes ou de rythmes antérieurs. ${ }^{26}$ Le fait est que cet alexandrin libéré n'est pas simplement un décalque du vers ancien mais le renouvellement d'une forme classique afin de lui permettre de répondre à des besoins nouveaux. Dans cette optique, Miron va assouplir encore davantage le moule qui lui est offert. Nous avons découvert un alexandrin libéré du compte strict des syllabes, fortement structuré en deux hémistiches par des pauses nettes à la césure et en fin de vers, alors que les coupes intermédiaires à la fin des première et troisième mesures sont beaucoup moins marquées. L'égalité des hémistiches constitue un élément primordial dans la perception du rythme alexandrin. On a dit que l'alexandrin classique devait sa pérennité à la multiplicité des symétries possibles autour de cet axe central de la césure au sixième pied. Je ne crois pas pour autant que Miron suive cette règle systématiquement. À côté de ses alexandrins coupés $6 / 6$, on observe des vers d'une douzaine de pieds qu'on ne peut pas couper $6 / 6$ mais $5 / 7$ ou $7 / 5$ et d'autres qui, me semble-til, perdraient à être forcés dans le moule 6/6 (par certaines apocopes) et qui gagnent au contraire, qui se révèlent, à être lus en deux hémistiches inégaux de cinq et sept pieds.

Regardons par exemple la fin du poème «Et l'amour même est atteint» (p. 52):

et j'entends ton rire de bijoux consumés

$\begin{array}{llllllllllllll}1 & 2 & 3 & / & 1 & 2 & / / & 1 & 2 & 3 & / & 1 & 2 & 3\end{array}$

dans le lit où déferlent les printemps du plaisir

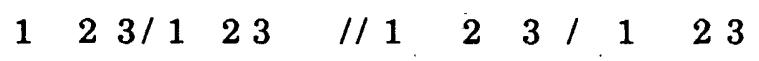

Il y aura toi et moi, et le cœur unanime

$\begin{array}{llllllllllll} & 2 & 3 / 1 & 2 & 3 & / / 1 & 2 & 3 & / & 1 & 2 & 3\end{array}$ 
je serai enfin dévêtu de ma fatigue

\section{$\begin{array}{lllllllllllllll}1 & 2 & 3 / 1 & 2 / / & 2 & 3 / 1 & 2 & 3 & 4\end{array}$}

À la suite de ce rythme régulier $3 / 3$ établi sur deux vers et demi, le dernier vers se veut indubitablement une rupture au niveau du rythme par sa division 5/7: avec le mot enfin, placé dans une mesure où il "manque" un pied, explose le soulagement final et, inversement, le pied «en trop " de dévêtu rend manifeste toute la difficulté du processus. On comprend alors l'intérêt stylistique de cet alexandrin asymétrique que Miron multiplie:

-moi qui ai des yeux où ciel et mer s'influencent

$$
\begin{array}{lllllllllllll}
1 & 2 & 3 / 1 & 2 & / / & 1 & 2 & 3 & 4 & / & 1 & 23 & =
\end{array}
$$

(«La marche à l'amour ", p. 36)

nous comme empesés d'humiliation et de mort

$$
\begin{array}{lllllllllllll}
1 / 1 & 2 & 3 & 4 / / & 1 & 2 & 3 & 4 / 1 & 2 & 3 & =5 / 7
\end{array}
$$

("Les siècles de l'hiver", p. $51^{27}$ )

à ces pas semés dans les rues sans nord ni sud

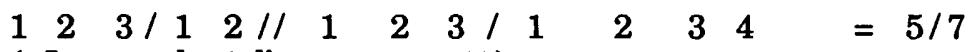

("La marche à l'amour ", p. 41)

Et quelle que soit la longueur qu'on attribue au vers ci-dessous, le dernier du "Damned Canuck» (p. 55), il ne me paraît pas souhaitable d'en faire un vers symétrique:

seulement les genoux seulement le ressaut pour dire

$$
\begin{array}{lllllllllllll}
1 & 2 / 1 & 2 & 3 & / / & 1 & 2 / 1 & 2 & 3 & 4 & 5=5 / 7
\end{array}
$$

Ce vers coupé $5 / 7$ (ou 7/5) contient d'ailleurs fréquemment une mesure centrale de deux (ou trois) pieds qui semble chevaucher la césure effacée et rétablit un élément de symétrie puisqu'on peut découper le vers 5/2/5. Ainsi:

quels ravages de toi ma belle dans le vide de toi

$$
\begin{array}{lllllllllllllllll}
1 & 2 & 3 & / & 1 & 2 / / & 1 & 2 & / / & 1 & 2 & 3 & / / & 1 & 2 & =5 / 2 / 5
\end{array}
$$

("Errant amour", p. 71)

27 Les hémistiches de cinq et sept pieds sont nombreux dans ce poème. 
fais-moi passer tout cabré tout empanaché

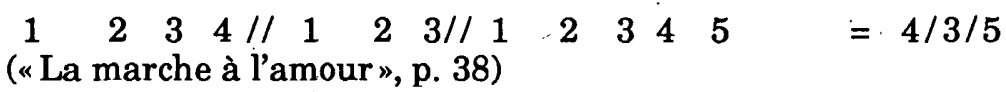

Ces derniers exemples nous rapprochent de deux autres procédés utilisés par Gaston Miron pour assouplir le rythme de son alexandrin et, plus profondément, je dirais, pour établir une indécision, un brouillage rythmiques dont nous verrons par la suite le profit. Ces deux nouveaux procédés sont l'enjambement sur la césure et le trimètre. À la différence du vers $5 / 7$, l'enjambement sur la césure exige, pour être perçu, que le rythme $6 / 6$ soit établi afin que la figure de l'enjambement apparaisse comme un écart par rapport à cette norme; autrement dit, il faut que le lecteur attende et enregistre une césure au sixième pied pour être surpris de voir le groupe syntaxique se poursuivre au-delà. Miron a su tirer de très beaux effets de ce procédé:

avec cette tache errante de chevreuil que tu as

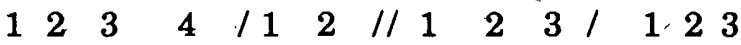

(“La marche à l'amour», p. 36)

en une femme aimée pour elle à cause de toi

$\begin{array}{lllllllllllll}1 & 2 & 3 & 4 & / 1 & 2 & / / & 1 & 2 & / 1 & 2 & 34\end{array}$

("Poème de séparation 2 ", p. $43^{28}$ )

Mais c'est le trimètre qui, de ce point de vue, a sa préférence. Miron l'utilise fréquemment - et exactement de la même manière que les poètes romantiques du XIX siècle - à la fin d'une strophe au rythme symétrique bien établi, afin d'obtenir un effet de rupture de rythme, de ralentissement et d'élargissement de la perspective:

le monde entier sera changé en toi et moi

$\begin{array}{lllllllllllllllllllllll}1 & 2 & 3 & 4 & / / & 1 & 2 & 3 & 4 / / 1 & 2 & 3 & 4\end{array}$

(«La marche à l'amoụr", p. 39)

et tu frissonnes à petit feu dans notre dos

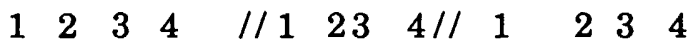

("Les siècles de l'hiver", p. 51)

28 Cette lecture avec enjambement sur la césure me semble plus expressive que la lecture en trimètre. 
les tourbillons des abattis de nos colères

$\begin{array}{llllllllllllll}1 & 2 & 3 & 4 & / / & 1 & 2 & 3 & 4 & / / & 1 & 2 & 34\end{array}$

("Recours didactique», p. 61)

comme le temps précieux et blond du sablier

$\begin{array}{lllllllllllll}1 & 2 & 3 & 4 & / / & 1 & 2 & 3 & 4 & / / & 1 & 2 & 34\end{array}$

("L'amour et le militant», p. 64)

J'ai la trentaine à brides abattues dans ma vie

\section{$\begin{array}{lllllllllllll}1 & 2 & 3 & 4 & / / 1 & 2 & 3 & 4 & 5 & / / & 1 & 2 & 3\end{array}$}

("Art poétique», p. 79)

Le poème "Compagnon des Amériques" offre des vers de longueurs variées qui sont beaucoup moins qu'à l'habitude établis dans le rythme binaire d'hémistiches à peu près égaux. Soit que les vers ne contiennent pas de césure nette, soit qu'ils présentent des coupes irrégulières: le rythme du poème en est bousculé et ce désordre traduit bien le message douloureux que nous livre Miron. À plusieurs reprises, un syntagme important occupe le centre du vers, faisant hé- siter la lecture entre enjambements sur la césure et trimètres approximatifs. Mais le vers le plus célèbre en est un trimètre (de onze pieds) indéniable:

mon Québec ma terre amère ma terre amande.

$\begin{array}{lllllllllllll}1 & 2 & 3 & / / & 1 & 2 & 3 & 4 & / / & 1 & 2 & 3 & 4\end{array}$
("Compagnon des Amériques", p. 56)

Cet alexandrin mironien, libéré des diverses façons que nous venons de voir, n'est évidemment pas le seul vers utilisé par le poète. Aucun autre vers, cependant, n'aura son importance métrique. Et il ne me paraît pas possible de lire les grands poèmes de Miron comme un assemblage de mètres différents ayant chacun leurs caractéristiques définies (ainsi qu'on le fait d'une fable de La Fontaine, par exemple). D'une part, la prononciation des $e$ atones introduit un élément d'incertitude dans l'établissement de la longueur - et donc du rythme - des vers. D'autre part, l'enchaînement des différents vers d'un poème (exception faite des poèmes réguliers) ne semble pas répondre à des règles précises de combinaisons hétérométriques. Le poème mironien est constitué d'un agencement de vers types flottant autour d'un modèle alexandrin et de vers plus longs ou plus brefs dont l'effet stylistique essentiel en est un de variation rythmique. Si l'on se penche maintenant sur la question de l'organisation de ce poème d'un point de vue métrique, le problème n'est donc pas, à mon avis, de relations hétérométriques mais de relations 
entre vers marqués métriquement (ceux que nous venons d'analyser) et vers moins définis métriquement. La réussite de Miron se situe précisément au niveau de l'unité qu'il a su réaliser entre ces vers de natures différentes; unité qui fait que, à la lecture, le poème se déroule avec une majesté, une régularité qu'on a pu qualifier d'épique ${ }^{29}$, en même temps qu'il se présente comme libéré de structures rythmiques trop étroites et capable des variations de rythme les plus modernes.

Techniquement parlant, le pari était difficile à tenir. En effet si l'alexandrin régulier s'impose trop fortement, les vers qui l'entourent, de neuf, onze ou treize pieds par exemple, seront entendus comme des vers faux et non comme des vers différents (cela a toujours été la difficulté d'utilisation des vers impairs). Si Miron réussit à combiner ces vers, c'est qu'à l'intérieur même de son alexandrin, il a déjà posé les variations de rythme que nous avons distinguées: un alexandrin trop régulièrement coupé $6 / 6$ instituerait une norme insurmontable pour les vers environnants; mais l'alexandrin mironien ne s'établit pas dans cette position hégémonique grâce d'une part au flottement créé par la présence des $e$ atones et grâce d'autre part à sa variété interne (alexandrins $5 / 7,4 / 4 / 4$, et autres). C'est cette libération de l'alexandrin qui lui permet de cohabiter avec des vers moins structurés, de longueur approximativement égale, en donnant l'impression globale non pas de vers boiteux mais de vers flottant librement autour d'un principe de régularité alexandrin, trouvant dans ce principe leur équilibre sans pour autant y être enfermés:

Ma pauvre poésie en images de pauvres

$$
\begin{array}{lllllllll}
1 & 2 / & 1 & 234 / / 1 & 2 & 3 / 1 & 2 & 3 & =6 / 6
\end{array}
$$

avec tes efforts les yeux sortis de l'histoire
1
$345 / / 1$
$2 / 12$
45
$=5 / 7$

avec tes efforts de collier au cou des délires

$\begin{array}{lllllllllllllll}1 & 2 & 3 & 4 & 5 & / / & 1 & 2 & 3 / & 1 & 2 & 3 & 45, & =5 / 8\end{array}$

ma pauvre poésie dans tes nippes de famille

$$
\begin{array}{lllllllllll}
1 & 2 / 1 & 234 / / & 1 & 2 & 3 & / & 1 & 2 & 3
\end{array}, \quad=6 / 6
$$

de quel front tu harangues tes frères humiliés
12
$3 / 1$
23
// 1
$23 / 123$
$=6 / 5$

29 G.-André Vachon, "Gaston Miron, ou l'invention de la substance», l'Homme rapaillé, édition P.U.M., p. 145. 
de quel droit tu vociferes ton sort avec eux

$\begin{array}{lllllllllll}1 & 2 & 3 / 1 & 2 & 34 & / / & 1 & 2 / 1 & 2 & 3 & =7 / 5\end{array}$

À lire ce début du poème "La pauvreté anthropos" (p. 76), on peut discuter les apocopes et les maintiens de $e$ atones que je propose, mais me semble indiscutable l'impression de régularité rythmique donnée par le texte. Et on découvre à l'analyse que cette régularité n'est pas celle d'un mètre exact mais qu'elle résulte de ce que j'appelle le «flottement» du vers autour de l'alexandrin.

$\mathrm{Au}$ niveau global du poème, ce flottement métrique est donc le résultat de trois flottements distincts: le premier provoqué par la présence des $e$ atones non prononcés, le second par les variations rythmiques internes au vers, le troisième par la juxtaposition de vers de longueurs diverses. On voit maintenant que l'empêchement d'un de ces types de flottement (en prononçant tous les $e$ atones, en refusant les coupes impaires $5 / 7$, en tentant de lire des vers impairs comme des vers pairs) ne ferait que détruire cette unité rythmique du poème: l'effet de fondu musical, de dégradés constants étant remplacé par un rythme heurté et mécanique. Ceci d'ailleurs est particulièrement vrai pour les poèmes riches en $e$ atones. La distinction que je propose entre $e$ "métriques" (comptés dans l'établissement des mesures) et $e$ "non métriques" (non prononcés et non comptés) ne peut masquer le fait qu'il n'est pas possible, à notre époque, de prononcer les $e$ maintenus ainsi qu'on le faisait dans les siècles passés. La langue a trop évolué: ces $e$ ont une valeur vocalique amoindrie. Néanmoins, leur présence, résultat d'une lecture intermédiaire entre l'artifice du maintien constant $\operatorname{des} e$ et la familiarité de leur apocope systématique, accroît la douceur musicale du vers (en réduisant le heurt des consonnes) et favorise la régularité rythmique (en introduisant le flottement dans chaque mesure). Des poèmes assez réguliers et structurés en quatrains comme "Jeune fille" (p. 34) ou "Frêle frileuse" (p. 66), particulièrement "féminins", y trouvent leur équilibre mélodique. Les deux "Poèmes de séparation", au rythme beaucoup plus varié et d'une mélodie certainement moins féminine, n'en constituent pas moins, eux aussi, de très beaux exemples de ce flottement autour de l'alexandrin. Mais ici l'éventail de vers est plus étendu et le poète a su tirer profit de plusieurs enjambements et rejets:

Tu fus quelques nuits d'amour en mes bras

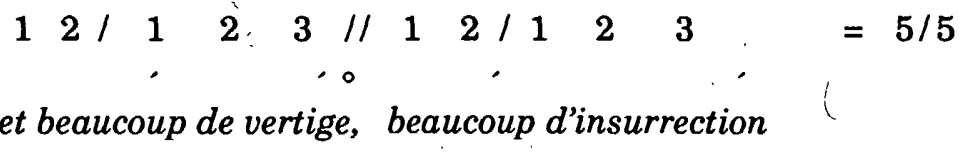

$\begin{array}{llllllllllllllll}1 & 2 & 3 & / & 1 & 2 & 3 & / / & 1 & 2 & / & 1 & 2 & 3 & 4\end{array}=6 / 6$


même après tant d'années de mer entre nous
$1 / 1$
3
$\begin{array}{llllllll}4 & 5 & / / & 1 & 2 / & 1 & 2 & 3\end{array}$
$=6 / 5$

à chaque aube il est dur de ne plus t'aimer

$\begin{array}{llllllllllllll}1 & 2 & 3 & / 1 & 2 & 3 / / & 1 & 2 & 3 . / & 1 & 2 & 2 & =6 / 5\end{array}$

parfois dans la foule, surgit l'éclair d'un visage
$12 / 1$
2
$/ / 12 / 12$
$3 \quad 45$
$=5 / 7$

blanc comme fut naguère le tien dans ma tourmente.
$1 / 1$
$\begin{array}{llllllll}2 & 3 & 4 & / / & 1 & 2 & \text { / } & 1\end{array}$
23
$4=5 / 6$
("Poème de séparation 2 ", p. $43^{30}$ )

À l'intérieur de cette homogénéité créée par le flottement métrique, on peut distinguer des éléments de diversification et des éléments d'unification. Parmi les éléments de diversification, il faut s'arrêter aux vers nettement plus brefs ou plus longs que la moyenne. Les vers très brefs me semblent avoir une fonction identique au rejet; groupés, comme dans "La marche à l'amour", ils équivalent à des vers longs dont le découpage interne serait mis en évidence par des pauses prolongées. En conséquence, les effets de rupture rythmique sont plutôt obtenus avec des vers de huit ou neuf pieds d'une part et des vers de quatorze à seize pieds d'autre part. Parmi ces vers très longs, certains se développent avec l'aisance et le naturel d'un mètre composé de trois parties équilibrées; "Héritage de la tristesse» constitue un bon exemple de poème en vers longs, en voici le début:

Il est triste et pêle-mêle dans les étoiles tombées

livide, muet, núlle part et effaré, vaste fantôme

il est ce pays seul avec lui-même et neiges et rocs

(p. 49)

Mais d'autres de ces vers m'apparaissent plutôt des vers types allongés d'une mesure excédentaire ainsi mise en relief:

j'ai su qu'une espérance soulevait ce monde jusqu'ici

$$
\begin{array}{llllllllllllllll}
1 & 2 / & 1 & 2 & 3 & 4 & / / & 1 & 2 & 3 / & 1 & 2 & / / & 1 & 2 & 3=6 / 5 / 3
\end{array}
$$

("Recours didactique", p. 61)

"Dans les lointains" (p. 77) présente ainsi, au sein de deux quatrains assez réguliers, ces deux longs vers où les mots frileux et

30 Vers 1: enjambement sur la césure; vers 3: enjambement sur la césure; vers 4: pourrait se lire $3 / 3 / 5$; vers 6 : rejet de blanc, enjambement sur la césure. 
natal sont placés en évidence, comme entre deux tirets, par l'enjambement sur la césure et leur nombre excédentaire:

avec les maigres mots frileux de mes héritages

$\begin{array}{llllllllllllll}1 & 2 & 3 & 4 & 5 & 6 / / & 1 & 2 & / / & 1 & 2 & 345\end{array}, \quad=6 / 2 / 5$

avec la pauvreté natale de ma pensée rocheuse
12

4
$56 / / 1$
$12 / / 1$

$\begin{array}{lllll}2 & 3 & 4 / & 1 & 2\end{array}$
$=6 / 2 / 6$

Un instrument plus subtil de variation rythmique est l'hendécasyllabe. La plupart du temps, le vers de onze pieds apparait simplement comme un flottement par rapport à l'alexandrin libéré. Mais dans un certain nombre de cas, il constitue au contraire un écart net, un nouveau mètre qu'on pourrait définir comme un alexandrin réduit. G.-A. Vachon l'avait déjà remarqué: Miron adopte souvent un mètre ample, asymétrique et régulier tout à la fois, adaptation, me semble-t-il, du vieux décasyllabe épique (4-6), dont il fait un alexandrin prolongé (6-8) ou diminué (5-6). ${ }^{31}$ A la différence de Vachon, je crois toutefois que le vers mironien trouve son origine dans l'alexandrin et non dans le décasyllabe épique; et que le vers de onze pieds est une rupture par rapport à l'alexandrin. Prenons par exemple les derniers vers de «Jeune fille» (p. 34.):

je parle de ces choses qui nous furent volées

$\begin{array}{llllllllllll}1 & 2 / 1 & 2 & 3 & 4 & / / & 1 & 2 & 3 / 1 & 23 & =6 / 6\end{array}$

mais les voudra la mort plus que l'ombre légère

$1234 / 1,2 / / 12,25 / 123=6 / 6$

nous serons tous deux allongés comme un couple

$\begin{array}{llllllll}1 & 23 / & 2 & 2 / 1 & 2 & 3 / 1 & 2 & 3\end{array}=5 / 6$

enfin heureux dans la mémoire de mes poèmes

$\begin{array}{llllllllllllll}1 & 2 & 3 & 4 & / / & 1 & 2 & 3 & 4 & / / & 1 & 2 & 34 & =4 / 4 / 4\end{array}$

Dans ce quatrain au rythme alexandrin très régulier s'élargissant, à la manière de Victor Hugo, sur un trimètre final, il aurait été facile à l'auteur d'ajouter un monosyllabe au troisième vers (nous serons tous les deux) afin d'obtenir un dodécasyllabe semblable aux précédents. L'effet de rupture est assez net. Si l'on compare ce troisième vers à d'autres cas identiques:

31 G.-A. Vachon, loc. cit., p. 145. 
la marche à l'amour s'ébruite en un voilier

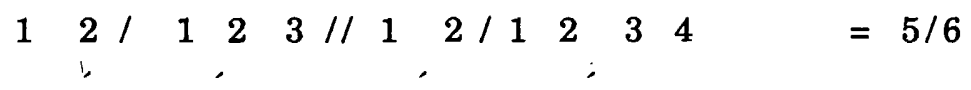

de pas voletant par les lacs de portage

$\begin{array}{llllllllllllll}1 & 2 / 1 & 2 & 3 & / / & 1 & 2 & 3 & / 1 & 2 & 3 & =5 / 6\end{array}$

de «La marche à l'amour» (édition Maspero, p. 40), ou de «L'amour et le militant» (édition P.U.M., p. 64):

\section{Quand je te retrouve après les camarades}

$$
\begin{array}{llllllllllll}
1 & 2 & 3 & 4 & 5 & / / 1 & 2 & 3 & 4 & 5 & 6 & =5 / 6
\end{array}
$$

on s'aperçoit que ce pied "manquant" donne un caractère spécifique à cet hendécasyllabe: c'est le vers qui brise le ronronnement satisfait de l'alexandrin, qui évite la somnolence causée par un rythme trop régulier et place un hémistiche en évidence grâce à ce blanc, à cette absence comparable à un silence en musique. Dans certains autres poèmes, enfin, le vers de onze pieds, par sa fréquence, pourra apparaitre aussi important, sinon plus, que l'alexandrin. Ainsi, dans la seconde partie de "La marche à l'amour" ( $t u$ viendras tout ensoleillée d'existence, p. 36), le rythme me semble s'amorcer autour de onze grâce à des hendécasyllabes de coupes variées, avant de s'amplifier légèrement. Et je fais la même constatation devant certains passages de Courtepointes comme "Il fait un temps fou " (Courtepointes, p. $37{ }^{32}$ ).

Derniers procédés de diversification rythmique que je voudrais mentionner, il s'agit des diverses figures (rejet, contre-rejet, enjambement, contre-enjambement) dont la définition commune est d'être une discordance entre le rythme de la phrase (déterminé par le découpage syntaxique) et le rythme métrique (déterminé par le découpage en vers). De même que dans le cas de l'enjambement sur la césure que nous avons déjà analysé, ces différents enjambements sont enregistrés en tant que figures dans la mesure où la fin du vers est normalement perçue et qu'ils sont entendus comme des débordements syntaxiques par-dessus cette limite métrique. Si les effets obtenus par Miron dans son utilisation des enjambements et rejets sont à ce point saisissants, c'est qu'il manie ces figures - avec grand art - dans le cadre de poèmes en vers nettement structurés ${ }^{33}$. Comme toujours, l'écart est d'autant plus apparent que la norme est

32 Poème repris en tête de «La marche à l'amour» dans l'édition Maspero, ce qui, du point de vue du rythme, est significatif de l'importance de l'hendécasyllabe dans cette suite.

33 "Avec töi" (p. 44) est un exemple de poème entier constamment bousculé dans son rythme par rejets, enjambements et parenthèses. 
sévère. La fonction du rejet et du contre-rejet (que Miron privilégie) est de fixer l'attention sur le mot ainsi isolé et de le faire résonner. Parmi les belles réussites de l'Homme rapaillé, on peụt remarquer:

de nouveau je m'avance vers toi, amour, je te demande passage, amour je te demande demeure

("Après et plus tard", p. 73)

cependant que la beauté aurifêre du froid t'auréole et comme nous dans la mort te sertit.

("Les années de déréliction", p. 82)

Et voici des exemples de contre-rejets:

j'ai un corps en mottes de braise où griffe un mal fluide de glace vive en ma substance ("Poème de séparation 2 ", p. 43)

déambulant dans un orbe calfeutré, les larmes poussent comme de l'herbe dans mes yeux ("Monologues de l'aliénation délirante», p. 58)

je voudrais m'enfoncer dans la mort nuit de métal enfin me perdre évanescent, me perdre dans la fascination de l'hébétude multiple (p. 58)

Ces procédés de diversification du rythme n'altèrent en rien, néanmoins, le sentiment prépondérant d'unité globale, de cohésion interne qui pénètre le lecteur traversant l'Homme rapaillé. Les poèmes de Gaston Miron s'écoulent comme les flots aux reflets changeants d'un fleuve unique impressionnant par sa puissance. Ampleur et structuration de la phrase mironienne, intensité sémantique et force particulière des verbes actifs toujours dominants, violence mélodique soutenue par des allitérations expressives: on a déjà distingué les multiples caractéristiques de cette puissance lyrique. Mais ce flot intense est contrôlé par la régulation rythmique que j'ai tenté de définir comme un flottement autour de l'alexandrin. Analysant l'énoncé mironien, Pierre Nepveu ${ }^{34}$ note sa complexité, -sa sinuosité ainsi que ses instruments d'organisation: il signale plusieurs types de répétitions (anaphores, refrains, compléments introduits par la même préposition, etc.). Ces répétitions constituent un autre niveau de contrôle de ce flot du discours. D'ailleurs le rythme, dans sa définition la plus simple, est un effet de répétition. Et la prépondérance de l'alexandrin libéré que nous avons étudiée, est la répétition systématique d'une certaine structure rythmique.

Mais nous avons vu aussi que ce flot mironien ne pouvait être contenu entre des digues trop étroites et qu'il débordait en une variété de

34 Pierre Nepveu, op. cit., p. 134 sq. 
vers. Cependant cette variété peut à son tour, je crois, être ramenée à un nouveau type de répétition. Si on décompose méthodiquement un poème hétérométrique de l'Homme rapaillé en ses hémistiches, les chiffres laissent voir, derrière la multiplicité des mètres, certaines constantes numériques. Prenons le début de "La marche à l'amour» (p. 36):

Tu as les yeux pers des champs de rosées

$12 / 1,2 \quad 3 / / 1 \quad 2 / 123 \quad=5 / 5$

tu as des yeux d'aventure et d'années-lumière

$\begin{array}{llllllllllllll}12 & 3 & 4 & / & 1 & 2 & 3 & / / 1 & 2 & 3 / 1 & 2\end{array},=7 / 5$

la douceur du fond des brises au mois de mai

$\begin{array}{lllllllllllllll}1 & 2 & 3 & / & 1 & 2 & 3 & 4 & / / & 1 & 2 & / & 1 & 2 & =\end{array}$

pour les accompagnements de ma vie en friche

$$
\begin{array}{cccccccccccccc}
1 & 2 & 3 & 4 & 5 & 6 & 7 & / / & 1 & 2 & 3 / 1 & 2 & =7 / 5
\end{array}
$$

avec cette chaleur d'oiseau à ton corps craintif
123
$45 / 1$
$2 / / 1$
$3 \quad / \quad 12$
$=7 / 5$

On constate une certaine diversité du rythme, diversité qui se poursuivra tout au long du poème; mais cette diversité est constituée par les combinaisons d'un nombre réduit d'hémistiches (ici les hémistiches de cinq et sept pieds) et certains d'entre eux (dans ce poème l'hémistiche de cinq) reviennent de manière récurrente. L'hypothèse que je propose sous le nom de "constante métrique", est que cet hémistiche (et plus généralement toute mesure ou tout groupe de mesures systématiquement récurrents) établit une relation d'identité entre des vers différents et par là assure lá cohésion rythmique d'un poème. Cette constante 5 serait donc ici, et ailleurs dans l'œuvre de Miron, l'élément rythmique qui permettrait la cohabitation dans un même texte de vers de dix $(5 / 5)$, onze $(5 / 6)$, douze $(5 / 7)$ et treize $(5 / 8)$ pieds. Et la juxtaposition de l'alexandrin coupé $5 / 7$ avec celui coupé $6 / 6$ permettrait le passage de ces vers «impairs" (intérieurement) aux vers "pairs " plus classiques (comme le décasyllabe 4/6). Si on généralise cette hypothèse, on pourrait voir dans la constante métrique l'unité rythmique fondamentale d'une œuvre poétique. Et c'est peut-être en découvrant que son vers naturel n'était pas l'alexandrin régulier 6/6 mais ce vers nouveau flottant autour de onze ou douze pieds divisé en deux hémistiches de cinq, six ou sept pieds, et donc gouverné par une constante métrique de 5 , que Miron a pu réaliser l'Homme rapaillé et faire entendre cette voix unique et si haute dans la poésie québécoise. 